\title{
Analysis of Three-Dimensional Damage Constitutive Model of Frozen Sand Based on the Influence of Intermediate Principal Stress and Temperature
}

\author{
Shilong Ma $\left(\mathbb{D}\right.$, Zhaoming Yao $\mathbb{D}$, Shuang Liu $\left(\mathbb{D}\right.$, and Xuan Pan $\mathbb{i D}^{\circ}$ \\ School of Civil Engineering and Architecture, Anhui University of Science and Technology, Huainan, Anhui 232001, China \\ Correspondence should be addressed to Zhaoming Yao; zhmyaoaust@126.com
}

Received 14 August 2020; Revised 9 September 2020; Accepted 14 September 2020; Published 26 September 2020

Academic Editor: Chong Xu

Copyright (c) 2020 Shilong Ma et al. This is an open access article distributed under the Creative Commons Attribution License, which permits unrestricted use, distribution, and reproduction in any medium, provided the original work is properly cited.

\begin{abstract}
To study the mechanical properties of frozen soil, it is necessary to understand the damage characteristics of frozen soil. Four types of three-dimensional indoor tests of frozen sand were carried out at $-5^{\circ} \mathrm{C},-10^{\circ} \mathrm{C}$, and $-15^{\circ} \mathrm{C}$ to study the mechanical damage properties. These include different stress path tests with the principal stress coefficients of $0,0.25,0.5$, and 0.75 while analyzing the entire failure process. First, the three-dimensional compression test of frozen sand was studied to analyze the influence of temperature and intermediate principal stress coefficient on the large principal stress of frozen soil. The damage cost of frozen sand under the influence of different temperatures and intermediate principal stress coefficients was also established. Second, using the characteristics of discreteness and randomness of the distribution of the microelements inside the frozen soil and assuming that the failure of the microelement of the frozen soil obeys the Weibull distribution, the Drucker-Prager strength criterion was used as the statistical distribution variable of the microelement of the frozen soil based on the strain equivalence hypothesis, statistical theory, and continuous damage mechanics. This allows for a constitutive model of frozen sand damage under the three-dimensional stress state to be established. Finally, the model parameter values through low-temperature threedimensional test data were able to be determined. This model allows for the physical meaning of Weibull distribution parameters $F_{0}$ and $m$ to be analyzed, and the distribution parameters with temperature and intermediate principal stress coefficient can be modified to obtain a modified frozen sand damage constitutive model. The results show that the modified damage constitutive model can simulate the entire process curve of the large principal stress-strain of frozen sand. It shows that the large principal stress of frozen sand increases with the increase of temperature and intermediate principal stress coefficient. Concurrently, the frozen sand damage constitutive model proposed in this paper can describe the deformation behavior of frozen soil under different temperature and stress paths and can be adapted to various other sediment types.
\end{abstract}

\section{Introduction}

Frozen soil is a relatively complex four-phase system composed of solid mineral particles, ice, liquid water, and gas [1]. Due to the existence of ice, frozen soil melts at high temperatures and freezes at low temperatures, which causes frost heave and thawing of the soil. The frost heave and thawing settlement of the soil will cause the accelerated development of randomly distributed pores, interfaces, and other defects in the interior, resulting in the formation of cracks, local shear bands, and overall damage to the soil structure. This, in turn, can cause irreversible plastic deformation. Furthermore, settlement due to frost heaving and thawing of the soil accelerates the damage of the soil structure. Frozen soil can be classified as either permafrost or seasonally frozen soil based upon its geographical location. Frozen soil accounts for about $24 \%$ of the Earth's land area [2,3]. In Northwest China, a large amount of frozen sand is distributed due to desertification, and the study of the mechanical properties of frozen sand plays a vital role in engineering construction in Northwest China. Due to this, the damage mechanics of frozen sand is an important research subject within frozen soil mechanics.

The current research on damage is mainly concerned with the damage mechanics analysis of frozen soil and rock. 
In order to understand the mechanical properties of frozen soil [4-8], many have conducted numerous frozen soil experiments [9-20]. Based on the continuous damage theory, it is believed that the generation, accumulation, and development of microcracks in frozen soil are caused by internal damage in frozen soil. Yao et al. [21, 22] established a general nonlinear strength theory applicable to concrete, rock, sand, and clay. Ren et al. [23] studied the microdamage mechanisms and damage constitutive model based on the uniaxial compression tests. The study showed that the damage mechanism can adequately describe the change in the stress-strain relationship. Qi and Ma [24] considered the influence of confining pressure, and a modified Mohr-Coulomb strength criterion was established to the study mechanical properties of the frozen soil. Lai et al. [25] proposed the relationship between the first principal stress and the third principal stress and established a nonlinear strength formula of frozen sand. Based on experimental results, Lai et al. [26] and Li et al. [27] proposed a statistical damage constitutive model of frozen clay suitable for high temperature and high ice content and used the Mohr-Coulomb strength criterion to determine if frozen soil elements were damaged during the freeze-thaw process. Tan et al. [28] established a damage constitutive model of cemented sand that considers the residual strength based on the assumptions of continuum damage mechanics and volume invariance. Also, [29-32] obtained many stressstrain relationship curves of rock damage and failure in the research of rock damage and failure mechanisms. Krajcinovic et al. [33], based on randomly distributed cracks in rock materials, combined continuous damage theory with statistical strength theory and proposed a statistical damage model, which provides a new method to study rock material constitutive models [34-36]. Based on the assumptions of strain equivalent and that the strength of rock microelements are affected by various random distributions (e.g., the Weibull distribution, power function distribution, normal distribution), a large number of statistical damage constitutive models have been proposed.

However, in actual engineering, the internal structure of frozen sand is more complicated than what is typically modeled. When only considering unilateral effects such as temperature or confining pressure, the damage of frozen sand cannot be studied adequately. Therefore, this article uses an artificial freezing method to conduct three-dimensional indoor tests of frozen sand under different conditions of low temperature and intermediate principal stress coefficient to obtain the stress-strain curve. Based on the test results, Hooke's law, and the damage mechanics theory, a three-dimensional damage constitutive model of frozen sand based on the Weibull random distribution is proposed. A modified three-dimensional damage constitutive model of frozen sand is then established to accommodate the variation law of the Weibull distribution parameters with the intermediate principal stress coefficient and temperature. This model better analyzes the mechanical failure characteristics of frozen sand and provides a theoretical basis for engineering design.

\section{Experimental Methods and Stress Paths}

2.1. Experimental Methods. The sand used in the test was Xiamen Aisiou standard sand. The samples were all remolded. The sand was air-dried, crushed, and sieved with a round-hole diameter of $0.1 \mathrm{~mm}$. The sand particle size distribution is shown in Figure 1. The sieved sand was then placed in a drying oven at $108^{\circ} \mathrm{C}$ and dried until a constant weight was reached, indicating no more moisture loss. The completely dried sand was configured according to the dry density of $1.95 \mathrm{~g} / \mathrm{cm}^{3}$ and the moisture content of $10 \%$. After the configuration was completed, the samples were then placed in a moisturizing dish for 24 hours.

After the 24 hours in the moisturizing dish, the sample was then molded using the layered vibrating compaction method. Petroleum jelly or antifreeze was evenly applied to the inner wall of the mold to prevent adhesion of the sample and allow for easier release. The walls were then covered with cling film or nanofilm to reduce water volatilization during freezing. The weighed sand was divided into five equal sections, placed in the mold individually, and compacted. Between each layer, the sample was shaved to avoid separation between layers. The mold size was $100 \mathrm{~mm} \times 100 \mathrm{~mm} \times 100 \mathrm{~mm}$. After the sample was placed into the mold, it was placed into a constant temperature control cabinet for 48 hours (or for 8 hours after overtemperature and for 24 hours at the target temperature) for demolding. The demolding stage occurred at the test temperature as much as possible.

The test used a ZSZ-2000 microcomputer-controlled True Triaxial Frozen Soil testing machine. It mainly consists of fixture (six rigid plates), loading system, cryogenic box, control cabinet, servo oil source, chiller, and computer. The major, intermediate, and minor principal stress can be freely converted in three directions. The structure diagram of the testing machine is shown in Figure 2 [37]. To ensure a constant temperature throughout the experiment, the testing machine contains a temperature control cabinet. The desired temperature was set on the temperature control cabinet and allowed to stabilize before a frozen, demolded sample was loaded into the device for 12 hours before the start of the test. Pressure was applied in three directions to start the consolidation of the experiment at this point and recorded throughout. The consolidation pressure was $1 \mathrm{MPa}$. After the consolidation was complete, stress loading control was performed, and the confining pressure was kept frozen according to the test requirements. The stress loading rate was $10 \mathrm{~N}-20 \mathrm{kN} / \mathrm{s}$. An example of before and after the test is shown in Figure 3.

2.2. Stress Path. In order to express the magnitude of the medium principal stress in the general stress state, the commonly used Bischoff's constant $b$ [38] (medium principal stress coefficient) is a parameter that reflects the relative magnitude of the intermediate principal stress $\sigma_{2}$ and the large principal stress $\sigma_{1}$ and the small principal stress $\sigma_{3}$. These can be as expressed as

$$
b=\frac{\sigma_{2}-\sigma_{3}}{\sigma_{1}-\sigma_{3}} .
$$




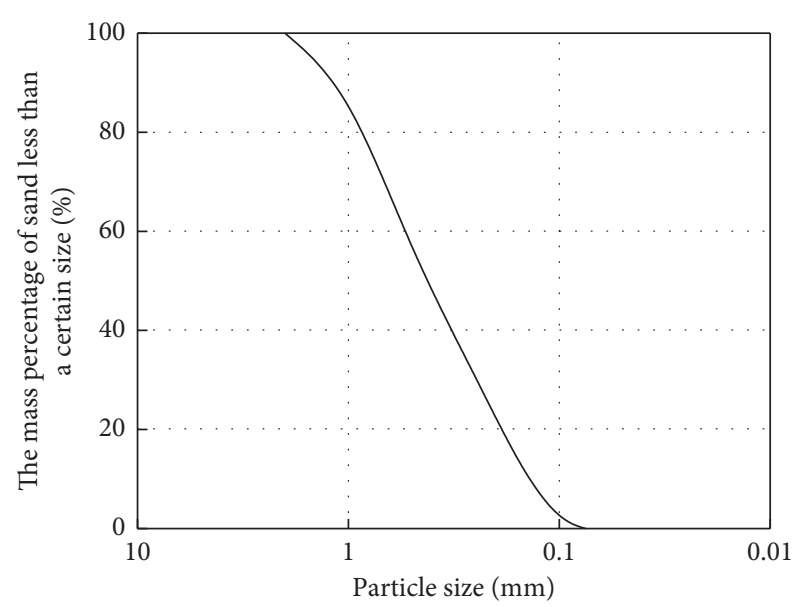

Figure 1: Grain distribution curve of samples.

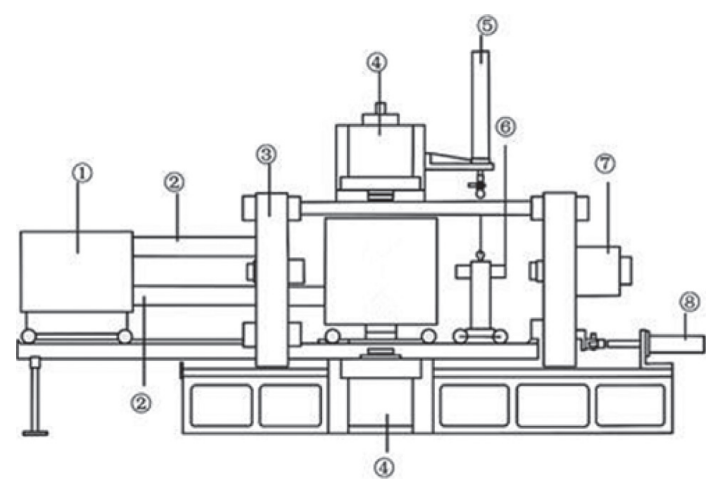

FIGURE 2: Setup diagram of artificially frozen soil true triaxial testing machine. (1) Temperature control cabinet. (2) Inlet air duct, return air duct. (3) Floating loading frame in $y$ direction. (4) $Z$ direction loading cylinder. (5) Lifting device for extension rod in $y$ direction. (6) Lengthening device in $y$ direction. (7) Load cylinder in $y$ direction. (8) Loading frame pulling device in $y$ direction.

From the previous equation,

$$
\sigma_{2}-\sigma_{3}=b\left(\sigma_{1}-\sigma_{3}\right) .
$$

If $\sigma_{3}$ is unchanged, yet increasing $\sigma_{1}$ and $\sigma_{2}$ and maintaining a constant $b$, equation (2) can be derived as

$$
\mathrm{d} \sigma_{2}=b \mathrm{~d} \sigma_{1} .
$$

In the experiment, stress is first applied three-ways to $\sigma_{3}$ so that the sample is in a state where the stress is applied equally in all directions. A load is then applied to the large principal stress surface and the intermediate principal stress surface in equal proportions according to formula (3) to ensure that the principal stress coefficient and the small principal stress do not change throughout the experiment. This is the stress path of the equal $b$ loading test in this article. See Figure 4.

Under the conditions of $-5^{\circ} \mathrm{C},-10^{\circ} \mathrm{C}$, and $-15^{\circ} \mathrm{C}$, four true triaxial tests of frozen sand with medium principal stress coefficients of $0,0.25,0.50$, and 0.75 were carried out, and three parallel tests were carried out for each coefficient.
A total of 36 samples were run. The minimum principal stress $\sigma_{3}$ is $1 \mathrm{MPa}$.

\section{Test Results}

Figure 5 shows the relationship between $\left(\sigma_{1}-\sigma_{3}\right)$ and $\varepsilon_{1}$ measured by the equal- $b$ loading test of frozen sand at $-5^{\circ} \mathrm{C}$, $-10^{\circ} \mathrm{C},-15^{\circ} \mathrm{C}, \sigma_{3}=1 \mathrm{MPa}$, and $b$ was $0,0.25,0.5$, and 0.75 , respectively. Figures 6 and 7 are the measured $\varepsilon_{v}-\varepsilon_{1}, \sigma_{q}-\varepsilon_{q}$ relationships [39].

Figure 5 shows that the deviator stress-strain relationship of the artificially frozen sand can be roughly divided into four stages. (1) Compaction stage: this stage mainly occurs when the major principal strain is less than $1 \%$, which shows that the deviatoric stress-strain curves of the specimen under different stress paths are mostly overlapping. This is because, during the initial loading of the artificially frozen sand, the pores inside the sample compact, and the sand particles and ice crystal particles rearrange, which is usually suitable for bearing external loads. (2) Elastic stage: this stage is mainly manifested once the deviator stress-strain curve is nearly linear, and the sample undergoes elastic deformation. When the load is removed, the deformation can be completely restored. The elastic deformation stage of the specimens under different stress paths occurs when the major principal strain is typically less than $2 \%$. This is because a load is small and typically results in little to no internal damage or deformation within the frozen sand specimen. As the middle principal stress coefficient increases, the slope of the curve also increases, indicating that the elastic modulus increases with an increase in the middle principal stress coefficient. (3) Strain hardening stage: as the load increases, the sample transitions from the linear elastic deformation stage to the nonlinear deformation stage. The sample undergoes both plastic and elastic deformations, of which plastic deformation is the primary deformation. Compared with the elastic phase, the stress increase required to produce the same strain increase in the strain hardening phase is much less. The stress increase decreases with an increase in the amount of deformation; that is, as the stress increases, the strain rate gradually increases as well. Also, during this stage, due to the gradual increase in the load, the ice crystals and cement inside the sample are destroyed and microcracks appear internally, which reduces the ability of the sample to resist damage and causes the strain rate to increase. This stage is also an essential stage of damage to the sample. (4) Yield stage: in this stage, the change in the stress is small while the strain increases sharply. Under a small stress increase during the stage, the overall deformation of the sample will be great. At this time, the sample is damaged, so the boundary point between the strain hardening stage and the yield stage can be the critical point of specimen failure. When the principal stress coefficient is a constant value, the deviator stress-strain curve of frozen sand is similar to the previously reported results $[26,40]$. As the axial deformation increases, the stress-strain curve gradually transitions from the elastic stage to the elastoplastic stage. Confining pressure shows strain softening, and high confining pressure shows strain hardening. 


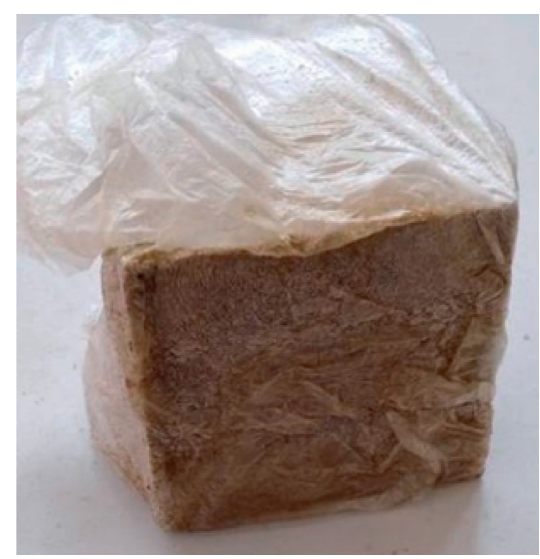

(a)

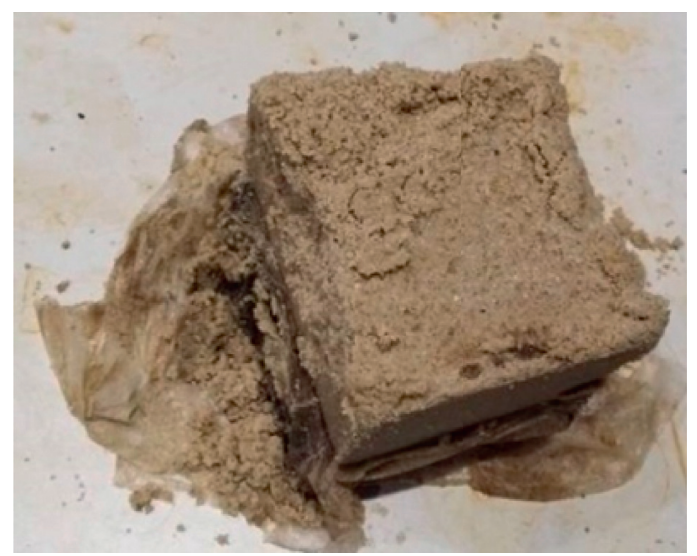

(b)

Figure 3: Sample (a) before and (b) after the test.

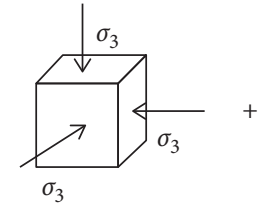

Load consolidation

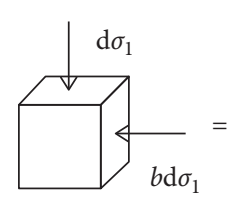

Load compression

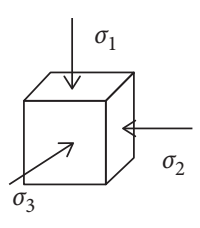

FIGURE 4: Sketch map of the loading path.

From Figure 5, when the temperature is the same, the deviator stress of the artificially frozen sand increases with the increase of the intermediate principal stress coefficient. When the large principal strains are the same, the curves from top to bottom are similar to the middle principal stress coefficients, and only individual curves are staggered. This shows that with an increase in the intermediate principal stress coefficient, the strength of frozen soil is enhanced. The soil increases its ability to resist deformation and the tendency of strain hardening as well. When the principal stress coefficients are the same, the deviator stress-strain relationship of the samples at various temperatures is relatively consistent. As the temperature decreases, though, the elastic modulus of frozen sand gradually increases.

Figure 6 shows the relationship curve between the strain of an artificially frozen sand body and large principal strain under different initial conditions. Overall, the changes in volume strain with varying initial conditions are similar. The overall sample first shows a trend of compression and then expansion. When the large principal strain is small, as the middle principal stress increases, the slope of the curve also increases. When the initial conditions are different, the large principal strain point at which the volume of artificially frozen sand changes from compression to expansion varies.

A frozen sand body is simultaneously affected by temperature and intermediate principal stress, which is mainly manifested when the temperature is constant and the intermediate principal stress coefficient is small. The sample is finally in an expanded state. When the principal stress coefficient is large, the sample is finally in a compressed state.
When the principal stress coefficient is constant, as the temperature decreases, the large principal strain corresponding to the constant volume point becomes larger and larger. At the same time, the volume strain inflection point also increases with an increase in the intermediate principal stress coefficient, simultaneously increasing the maximum compression value. It is shown in Figure 6 that the sample curve and the intermediate principal stress coefficient coincide.

When $b=0$, the sample often reaches the inflection point of volume strain when the major principal strain is $1 \%-3 \%$. When $b=0.75$, the sample often reaches the volume strain inflection point when the major principal strain is $3 \%-6 \%$. Simultaneously, as the axial strain reaches the inflection point of volume strain, the slope of the curve gradually decreases as the intermediate principal stress coefficient increases. As the intermediate principal stress coefficient increases, the increase in the intermediate principal stress limits the expansion of the soil, which causes the strain inflection point to move back, the slope decreases, and the final strain state is different. However, in [40], when the temperature is constant, the volume exhibits a phenomenon of body shrinkage and then body expansion under low confining pressure, while sediment body shrinkage occurs under high confining pressure. As in this article, when the temperature is constant, a change in the middle principal stress coefficient results in corresponding volume changes in the sand body.

3.1. Sand at Different Temperatures. Comparing the generalized shear stress-generalized shear strain curve (Figure 7), when the shear strain is small, the shear stress required to produce the unit shear strain is almost the same. When the shear strain is large, the shear stress required to produce a unit shear strain increases with the increase in intermediate principal stress. The sample is in a compacting stage early during loading, during which the pores and cracks in the sample are compressed and reduced. The particles and the ice cement are better arranged to bear the load, and the 


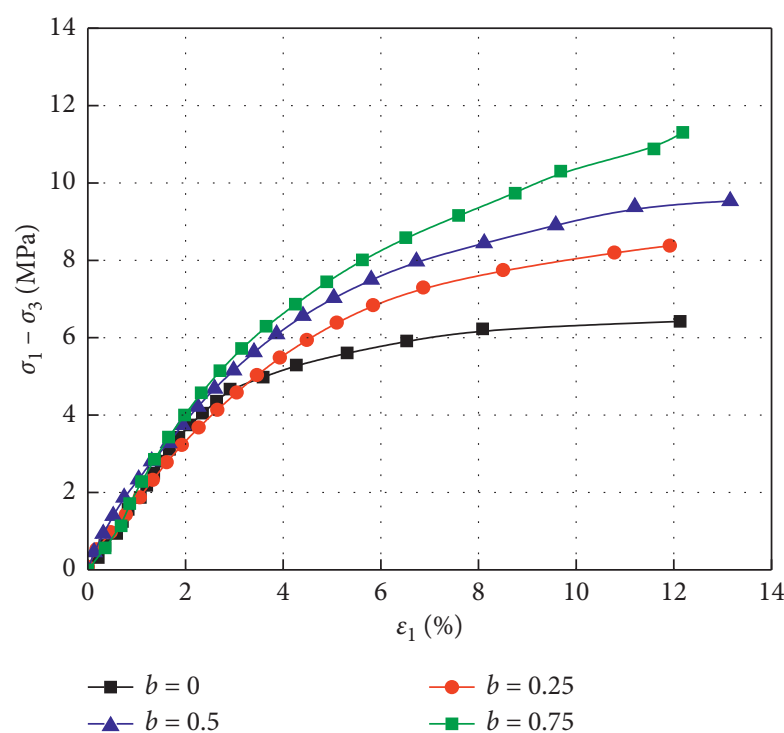

(a)

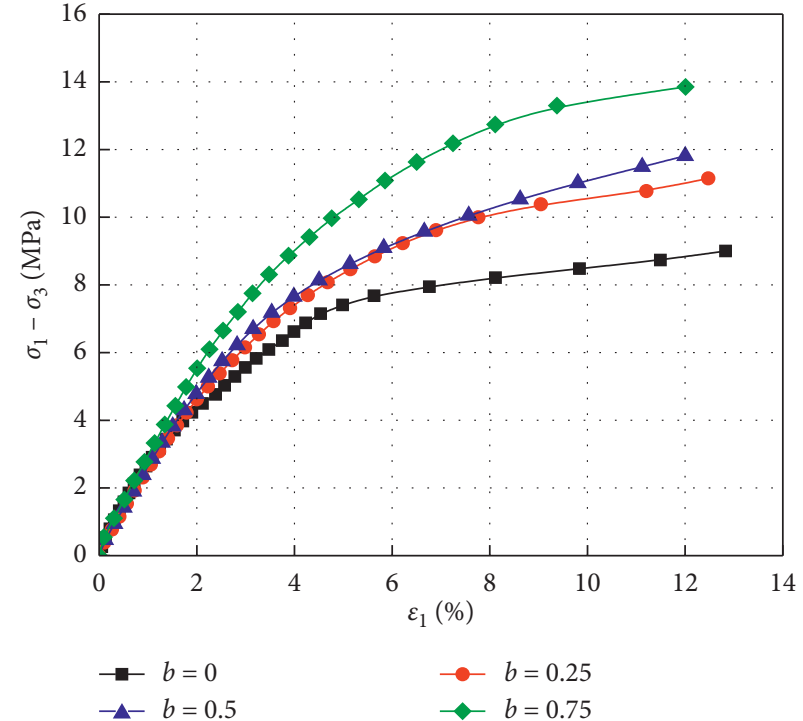

(b)

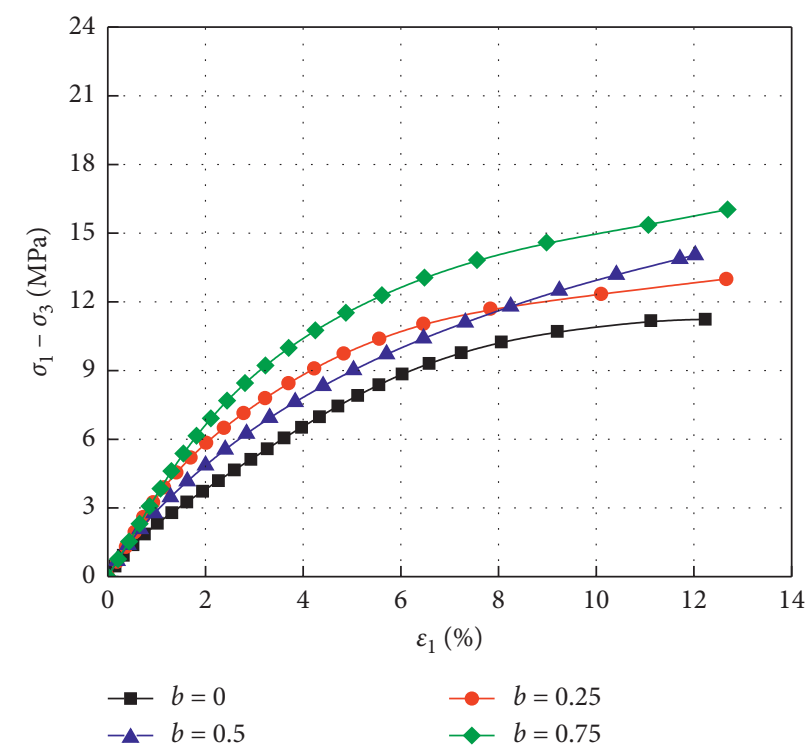

(c)

Figure 5: Curves of partial stress and large principal strain of frozen sand at different temperatures. (a) $-5^{\circ} \mathrm{C}$. (b) $-10^{\circ} \mathrm{C}$. (c) $-15^{\circ} \mathrm{C}$.

stress-strain relationship curve indicates hardening of the sample.

When the temperature is constant, an increase in the intermediate principal stress coefficient increases both the generalized shear stress and the generalized shear strain during the failure of frozen sand. This indicates that different stress loading paths have an effect on the resistance of soil to damage. When the principal stress coefficient is constant, as the temperature decreases, both the generalized shear stress and the generalized shear strain at the failure point increase. This is because as the temperature decreases, cohesion increases and the degree of cementation of ice and soil increases as well. The soil has a better bearing capacity and the ability to resist damage. In [41], a true triaxial test study on the influence of the intermediate principal stress coefficient and stress path on the shear characteristics of sand was carried out. The study shows that, with an increase in the intermediate principal stress coefficient, the shear strength of sand gradually increases, which is consistent with the test results in this paper.

\section{Damage Constitutive Model of Frozen Sand}

4.1. Damage Variables. Based on the strain equivalence hypothesis proposed by Lemaitre [10], the strain caused by the stress acting on a damaged material is equivalent to the strain caused by equivalent stress acting on a nondamaged material. This can be expressed as 


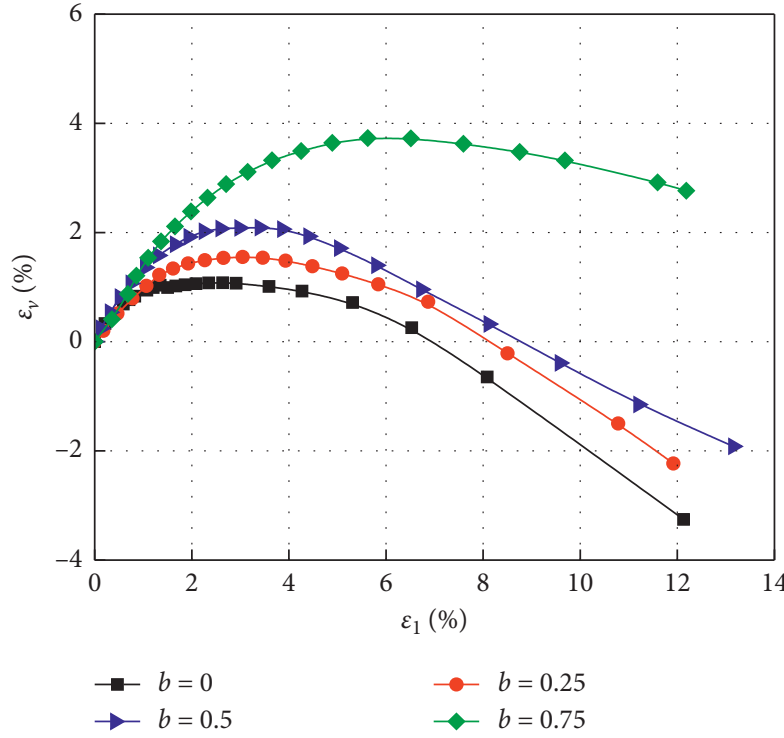

(a)

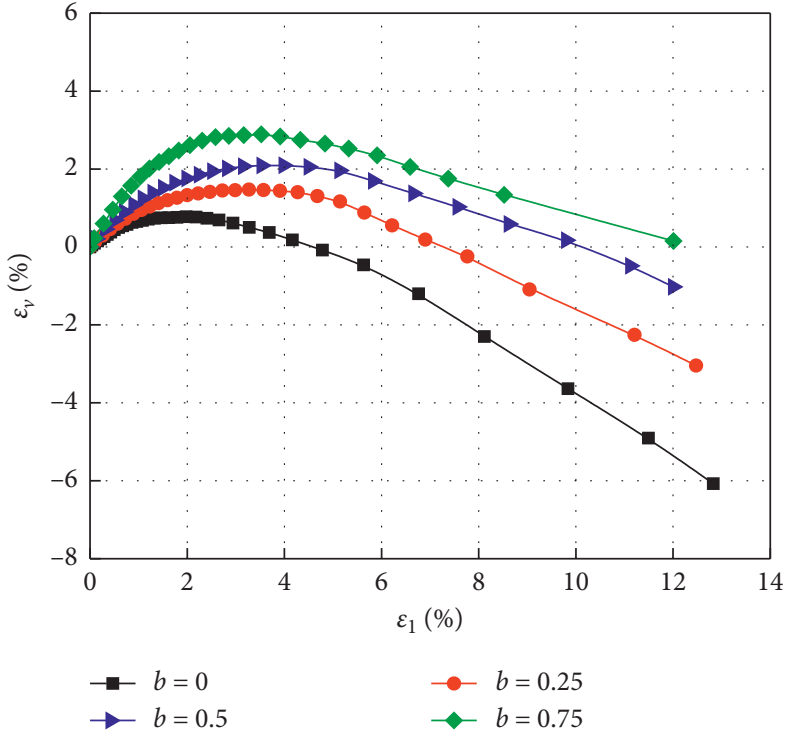

(b)

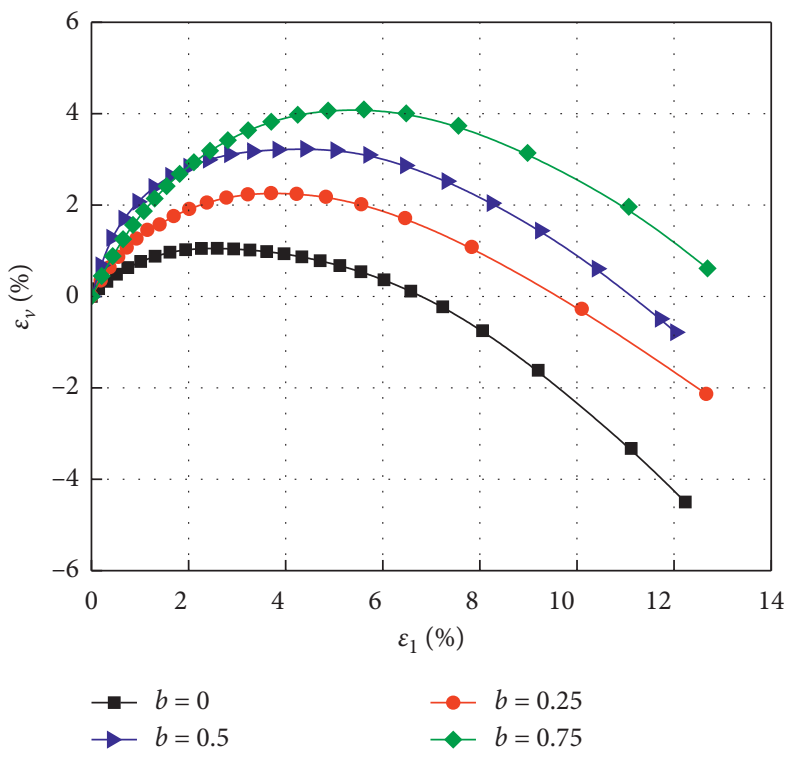

(c)

Figure 6: Curves of strain compared to large principal strain in frozen sand at different temperatures. (a) $-5^{\circ} \mathrm{C}$. (b) $-10^{\circ} \mathrm{C}$. (c) $-15^{\circ} \mathrm{C}$.

$$
\begin{aligned}
\sigma^{*} & =\frac{\sigma}{1-D}, \\
\varepsilon & =\frac{\sigma^{*}}{E}=\frac{\sigma}{E^{*}}=\frac{\sigma}{E(1-D)},
\end{aligned}
$$

where $E$ is the elastic modulus of the nondamaged material, $E^{*}$ is the elastic modulus of the damaged material, $\varepsilon$ is the strain, $D$ is the damage variable, $\sigma$ is the nominal stress, and $\sigma^{*}$ is the effective stress.

The microscopic view of the damage statistics variable is the ratio of the number of damaged cells to the total number of cells. The macroview is the ratio of the number of damaged cells to the total number. The expression is [28]

$$
D=\frac{N_{f}}{N}
$$

where $N_{f}$ is the number of damaged units and $N$ is the total number of units.

Supposing the probability of damage to the frozen soil microelements is $P(y)$, the number of microelements that have been destroyed in any interval $[F, F+D F]$ is $N P(y) \mathrm{dy}$. When the soil is loaded to a certain level $F$, the number of microelements destroyed can be determined by [30]

$$
N_{f}(F)=\int_{0}^{F} N P(y) \mathrm{d} y .
$$

The frozen soil damage variables are 


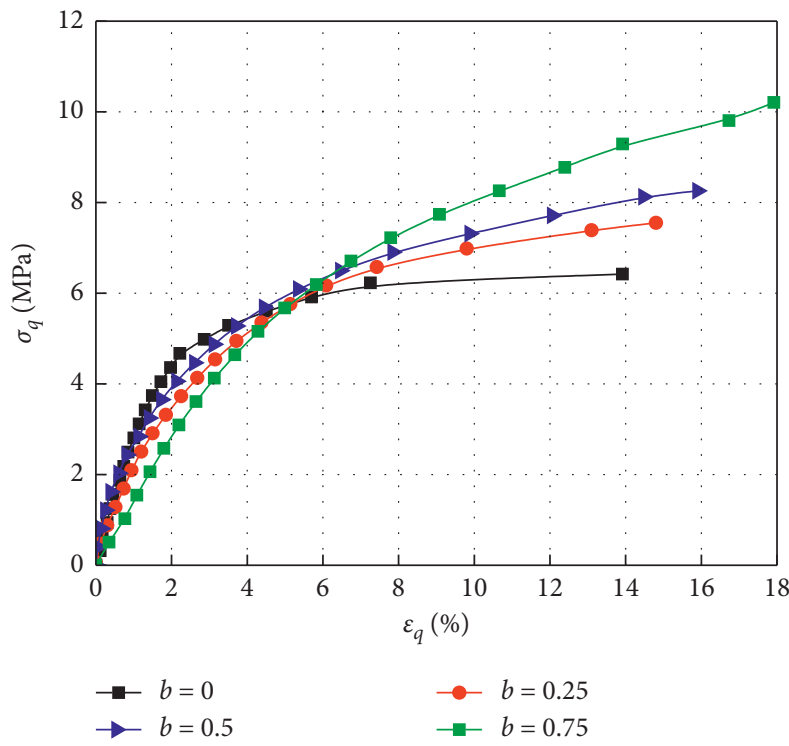

(a)

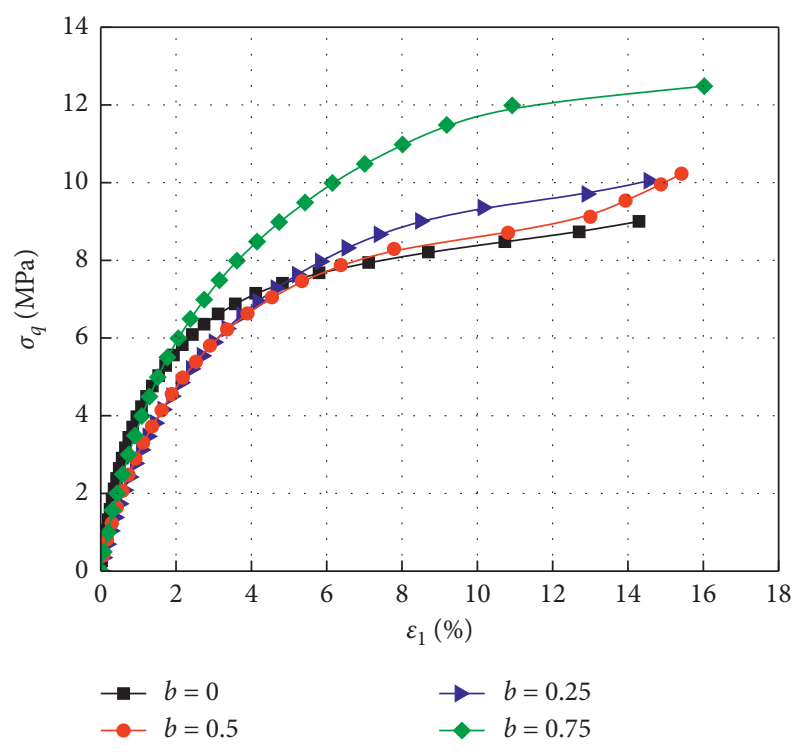

(b)

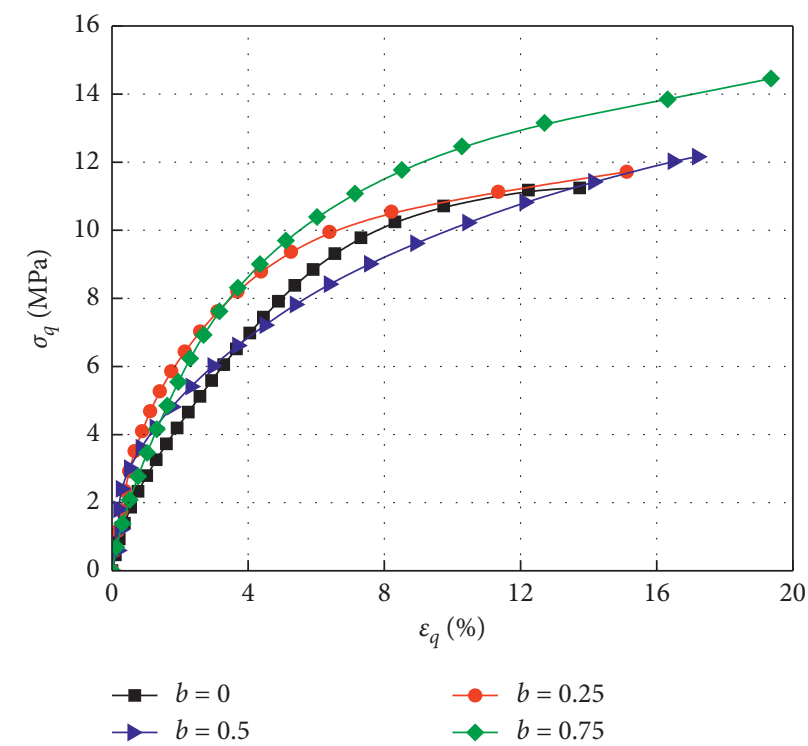

(c)

FiguRE 7: Relationship between generalized shear stress and generalized shear strain of frozen sand at different temperatures. (a) $-5^{\circ} \mathrm{C}$. (b) $-10^{\circ} \mathrm{C}$. (c) $-15^{\circ} \mathrm{C}$.

$$
D=\frac{N_{f}}{N}=\frac{\int_{0}^{F} N P(y) \mathrm{d} y}{N}=\int_{0}^{F} P(y) \mathrm{d} y .
$$

4.2. Triaxial Damage Model of Frozen Soil and Determination of Model Parameters. In the study of frozen soil mechanics, the manifestation of the failure criterion can be expressed by a general formula:

$$
f(\sigma)=K_{0},
$$

where $f(\sigma)$ is the yield criterion of frozen soil microelements related to the stress state and $K_{0}$ is a constant, a parameter that changes with the stress state, and is a constant related to the cohesion of the material and the angle of internal friction. If $f(\sigma) \geq K_{0}$, the frozen soil microelements have yielded or failed, and the relationship between $f(\sigma)$ and $K_{0}$ can be used to determine the state of frozen soil microelements [40].

$F=f(\sigma)$ can be used to indicate the strength of the microelements of frozen sand. The expression of the microelement strength of frozen sand based on the Drucker-Prager yield criterion is expressed as

$$
\begin{aligned}
& F=f(\sigma)=\alpha_{0} I_{1}+J_{2}^{1 / 2}, \\
& \alpha_{0}=\frac{\sin \varphi}{\sqrt{9+3(\sin \varphi)^{2}}},
\end{aligned}
$$




$$
\begin{aligned}
& I_{1}=\sigma_{1}^{*}+\sigma_{2}^{*}+\sigma_{3}^{*}, \\
& J_{2}=\frac{1}{6}\left[\left(\sigma_{1}^{*}-\sigma_{2}^{*}\right)^{2}+\left(\sigma_{2}^{*}-\sigma_{3}^{*}\right)^{2}+\left(\sigma_{1}^{*}-\sigma_{3}^{*}\right)^{2}\right],
\end{aligned}
$$

where $\varphi$ is the internal friction angle of the sand, $F\left(\sigma^{*}\right)$ is the random distribution variable of the microelement strength of the frozen soil, $\sigma_{1}^{*}, \sigma_{2}^{*}, \sigma_{3}^{*}$ represent the effective stresses of the frozen soil, $I_{1}$ is the first difference of the stress tensor variable, and $J_{2}$ is the second invariant of the stress deflection.

From Hooke's law and formula (4),

$$
\begin{gathered}
\varepsilon_{1}=\frac{\sigma_{1}^{*}-\mu\left(\sigma_{2}^{*}+\sigma_{3}^{*}\right)}{E}, \\
\sigma_{i}^{*}=\frac{\sigma_{i}}{1-D}, \quad i=1,2,3 .
\end{gathered}
$$

Thus,

$$
\begin{aligned}
I_{1} & =\frac{\left(\sigma_{1}+\sigma_{2}+\sigma_{3}\right) E \varepsilon_{1}}{\sigma_{1}-\mu\left(\sigma_{2}+\sigma_{3}\right)}, \\
\sqrt{J_{2}} & =\frac{E \varepsilon_{1} \sqrt{\left(\sigma_{1}-\sigma_{2}\right)^{2}+\left(\sigma_{2}-\sigma_{3}\right)^{2}+\left(\sigma_{1}-\sigma_{3}\right)^{2}}}{\left[\sigma_{1}-\mu\left(\sigma_{2}+\sigma_{3}\right)\right] \sqrt{6}} .
\end{aligned}
$$

From equations (10), (11), (16), and (17), the microelement strength of frozen soil expressed by the nominal stress can be obtained.

Assuming that the microelement intensity distribution of frozen sand obeys the Weibull random distribution, the probability density function expression of Weibull distribution is as follows [33-35]:

$$
P(F)=\frac{m}{F_{0}}\left(\frac{F}{F_{0}}\right)^{m-1} \exp \left[-\left(\frac{F}{F_{0}}\right)^{m}\right],
$$

where $P(F)$ is the random distribution function of the microelement strength of frozen soil and $m$ and $F_{0}$ are the distribution parameters.

Substituting formulas (7) into (17), we obtain

$$
D=\int_{0}^{F} P(y) \mathrm{d} y=1-\exp \left[-\left(\frac{F}{F_{0}}\right)^{m}\right] \text {. }
$$

Substituting the above equations into equations (14), (15), and (4), we can obtain the constitutive relationship of frozen sand damage based on the Weibull distribution [36]:

$$
\sigma_{1}=E \varepsilon_{1}(1-D)+\mu\left(\sigma_{2}+\sigma_{3}\right)=E \varepsilon_{1} \exp \left[-\left(\frac{F}{F_{0}}\right)^{m}\right]+\mu\left(\sigma_{2}+\sigma_{3}\right) .
$$

Simplifying and transforming formula (20) gives [29]

$$
\frac{\sigma_{1}-\mu\left(\sigma_{3}+\sigma_{2}\right)}{E \varepsilon_{1}}=\exp \left[-\left(\frac{F}{F_{0}}\right)^{m}\right] \text {. }
$$

Taking the logarithm of equation (21) on both sides gives

$$
-\ln \left(\frac{\sigma_{1}-\mu\left(\sigma_{3}+\sigma_{2}\right)}{E \varepsilon_{1}}\right)=\left(\frac{F}{F_{0}}\right)^{m}=\alpha F^{m} .
$$

It is further simplified into

$$
\ln \left[-\ln \left(\frac{\left(\sigma_{1}-\mu\left(\sigma_{3}+\sigma_{2}\right)\right)}{E \varepsilon_{1}}\right)\right]=\ln \alpha+m \ln F .
$$

Set the parameters in formula (23) to

$$
\begin{aligned}
& \alpha=\left(\frac{1}{F_{0}}\right)^{m}, \\
& Y=\ln \left[-\ln \left(\frac{\sigma_{1}-\mu\left(\sigma_{3}+\sigma_{2}\right)}{E \varepsilon_{1}}\right)\right], \\
& X=\ln F, \\
& d=\ln \alpha .
\end{aligned}
$$

From equations (23) to (27), equation (23) can be further simplified into

$$
Y=m X+d
$$

Linear regression provides the values of $m$ and $b$, and the value of $F_{0}$ can be obtained by

$$
F_{0}=\exp \left(-\frac{d}{m}\right)
$$

The values of the parameters $m$ and $F_{0}$ under different intermediate principal stress coefficients can be calculated using formulas (20) (29) (see Table 1). The obtained parameter values are then substituted into the true triaxial damage constitutive equation of frozen sand (formula (20)). The comparison chart of the theoretical curve and test curve under different principal stress coefficients is shown in Figure 8. The correlation coefficients of the experimental and theoretical curves at different temperatures and intermediate principal stress coefficients are shown in Table 2.

Analyzing Figure 8 and Table 2 shows that the model can fully reflect the entire process of frozen sand under loading. This reflects the fact that the strength of frozen sand is affected by temperature and the coefficient of intermediate principal stress. As the temperature decreases and the coefficient of intermediate principal stress increases, the strength of frozen sand increases. From the correlation coefficient in Table 2, the experimental curve and theoretical curve fit well. However, when $b=0.5$ and $b=0.75$, the theoretical curve and the experimental curve do not match after the large principal strain reaches a certain value, and there is a large error in the model. Therefore, a reasonable correction method is needed to fully reflect the true triaxial damage constitutive equation. 
TABLE 1: Fitting results of the Weibull parameters at different temperatures and medium principal stress coefficients.

\begin{tabular}{lccc}
\hline$T\left({ }^{\circ} \mathrm{C}\right)$ & $b$ & $F_{0}(\mathrm{MPa})$ & $m$ \\
\hline-5 & 0 & 4.437 & 0.329 \\
-5 & 0.25 & 0.9075 & 0.206 \\
-5 & 0.5 & 1.6487 & 0.24 \\
-5 & 0.75 & 0.26447 & 0.164 \\
-10 & 0 & 7.24 & 0.378 \\
-10 & 0.25 & 1.3868 & 0.214 \\
-10 & 0.5 & 2.3396 & 0.248 \\
-10 & 0.75 & 0.37157 & 0.167 \\
-15 & 0 & 1.3771 & 0.22 \\
-15 & 0.25 & 1.215 & 0.205 \\
-15 & 0.5 & 0.3945 & 0.177 \\
-15 & 0.75 & 0.28365 & 0.157 \\
\hline
\end{tabular}

\section{Modification of the Damage Constitutive Model of Frozen Sand}

5.1. Modification of Elastic Modulus of Frozen Sand. The elastic modulus is one of the essential parameters reflecting the mechanical properties of materials. It is defined as the stress value required to produce unit elastic deformation under an external load. The elastic modulus is divided into the static elastic modulus and dynamic elastic modulus. The static elastic modulus can be further divided into the tangent modulus, secant modulus, and chord modulus. The method of selecting the elastic modulus of frozen sand is the same as that of conventional soil, where the secant modulus is selected as the elastic modulus value of frozen soil. Therefore, the ratio of the corresponding axial strain to half of the uniaxial compressive strength is taken as the corresponding elastic modulus value [42].

Based on the deviatoric stress-strain curve at different temperatures (see Figure 5), the trend of change of the elastic modulus at different temperatures and intermediate principal stress levels is shown in Figure 9.

It can be seen from Figure 9 that with a decrease in the freezing temperature or an increase in the intermediate principal stress coefficient, the elastic modulus of frozen sand gradually increases. Through linear regression analysis, the elastic modulus is approximately linear with temperature and the principal stress coefficient with a correlation coefficient $R^{2}$ of 0.98 . The following linear equation is from fitting the above data:

$$
E=1489.089 b-91.225 T+217.355 \text {. }
$$

\subsection{Physical Meaning of the Weibull Distribution Parameters} $F_{0}$ and $m$. The test data in Table 1 and formula (20) are used to explain the physical meaning of parameters $F_{0}$ and $m$ in the frozen sand damage constitutive model, as shown in Figure 10.
From Figure 10, when the parameter $m$ is constant 0.25 , the peak value of the stress-strain curve of frozen sand increases with an increase in $F_{0}$. A larger $F_{0}$ results in a greater ability of the frozen sand to resist deformation and damage. The parameter $F_{0}$ reflects the strength characteristics of the frozen sand. When the parameter $F_{0}$ is constant 2.34, a larger parameter $m$ indicates a lesser ability of the frozen sand to resist deformation and failure and a more obvious brittleness of the soil. Therefore, parameter $m$ represents the ductility and brittleness characteristics of frozen sand. When $b=0$, this is similar to the previous research conclusions [40].

5.3. Correction of the Weibull Distribution Parameters $F_{0}$ and $m$. According to Table 1, the change in the Weibull distribution parameters $F_{0}$ and $m$ under the influence of different temperatures and intermediate principal stress coefficients are shown in Figure 11. When the temperature is constant, the parameter $m$ shows a downward trend as the middle principal stress coefficient increases (Figure 11(a)). When the principal stress coefficient is constant, parameter $m$ first increases and then decreases as the temperature decreases. When the temperature is constant, the parameter $F_{0}$ decreases as the middle principal stress coefficient increases, as shown in Figure 11(b). When the principal stress coefficient is constant, the parameter $F_{0}$ first increases and then decreases as the temperature decreases. From the above analysis, the Weibull distribution parameters $F_{0}$ and $m$ are affected by the temperature and the intermediate principal stress coefficient.

Based on this, the Weibull distribution parameters $F_{0}$ and $m$ were corrected using temperature and the intermediate principal stress coefficient. Through linear regression analysis, the Weibull distribution parameters $m$ and $F_{0}$ are approximately linearly related to the temperature and the intermediate principal stress coefficient. The following linear equations (31) and (32) are fitted by the above data:

$$
\begin{aligned}
& m=0.003 T-0.164 b+0.317 \\
& F_{0}=0.077 T-3.762 b+4.122 .
\end{aligned}
$$

Substituting formulas (30), (31), and (32) into (20) gives the modified true triaxial damage constitutive equation of frozen sand. The establishment of a constitutive model for simulating frozen sand damage is divided into two steps. The first step is to establish a damage constitutive model of frozen sand under different intermediate principal stresses. The second step consists of considering the influence of different intermediate principal stress coefficients on the damage constitutive model of frozen sand to modify the model. A statistical damage constitutive model that simulates the failure process of frozen sand can then be obtained: 


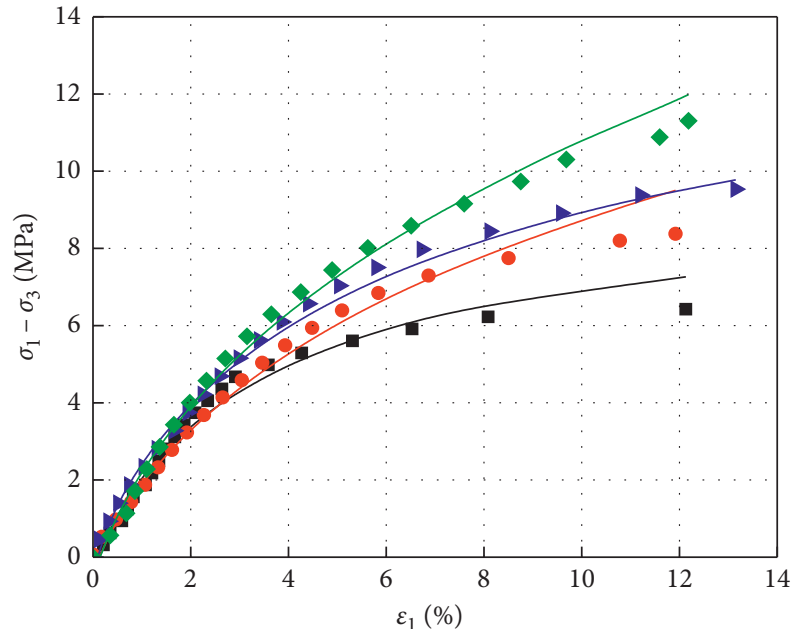

- $b=0$ Test curve - $b=0.25$ Test curve - $b=0.5$ Test curve $b=0.75$ Test curve

(a)

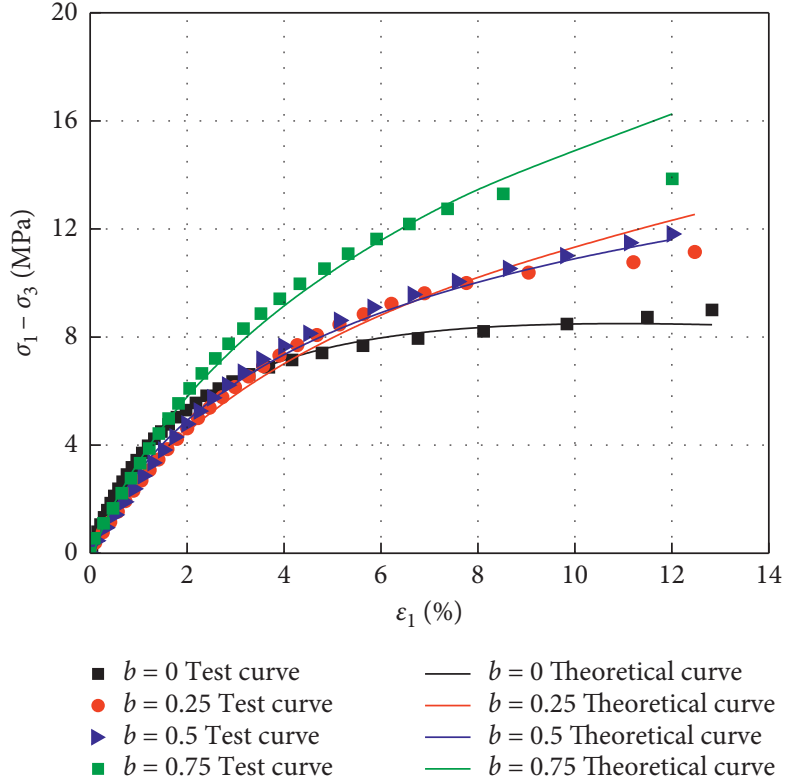

(b)

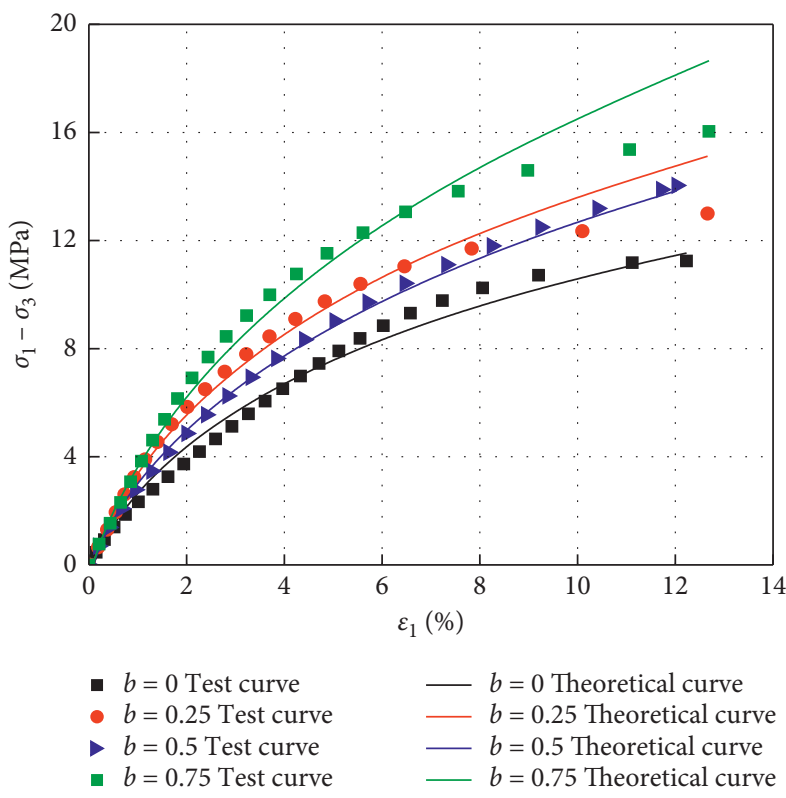

(c)

Figure 8: Theoretical and experimental curves of the true triaxial tests with varying $b$ values. (a) $-5^{\circ} \mathrm{C}$. (b) $-10^{\circ} \mathrm{C}$. (c) $-15^{\circ} \mathrm{C}$.

$\sigma_{1}=(1489.089 b-91.225 T+217.355) \varepsilon_{1} \exp \left[-\left(\frac{F}{0.077 T-3.762 b+4.122}\right)^{0.003 T-0.164 b+0.317}\right]+\mu\left(b \sigma_{1}+2 \sigma_{3}\left(1-\frac{b}{2}\right)\right)$

\section{Verification of the Model}

Using the experimental data and formula (33) in this paper, the frozen sand damage constitutive model proposed in this paper considering the influence of temperature and intermediate principal stress coefficient was verified, as shown in Figure 12.
The test curve in Figure 12 was analyzed and then revised to better reflect experimental results. Under different temperatures and intermediate principal stress coefficients, the revised theoretical curve fully reflects the failure characteristics of frozen sand under the true triaxial stress state and the fact that the strength of frozen sand changes with temperature and intermediate principal stress coefficients. Although there is a 
TABLE 2: Correlation coefficients of experimental and theoretical curves at different temperatures and intermediate principal stress coefficients.

\begin{tabular}{lcccccccccccc}
\hline$T\left({ }^{\circ} \mathrm{C}\right)$ & -5 & -5 & -5 & -5 & -10 & -10 & -10 & -10 & -15 & -15 & -15 & -15 \\
\hline$b$ & 0 & 0.25 & 0.5 & 0.75 & 0 & 0.25 & 0.5 & 0.75 & 0 & 0.25 & 0.5 & 0.75 \\
$R^{2}$ & 0.97 & 0.96 & 0.99 & 0.98 & 0.98 & 0.97 & 0.99 & 0.96 & 0.98 & 0.94 & 0.99 & 0.94 \\
\hline
\end{tabular}

$R^{2}$ in this table represents the correlation coefficient.

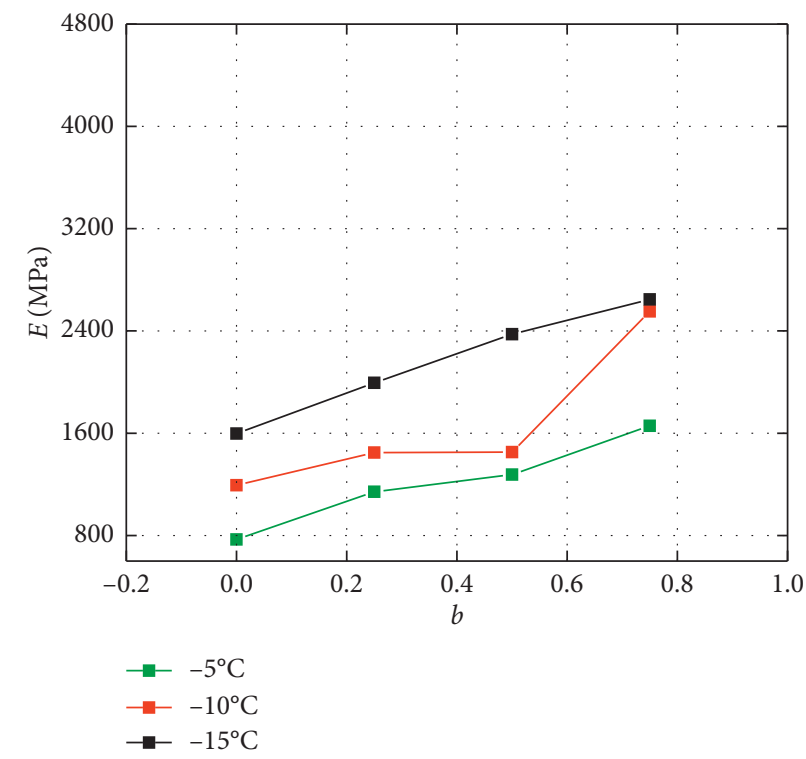

Figure 9: The relationship between the elastic modulus $E$ and the temperature $T$ and the intermediate principal stress coefficient $b$.

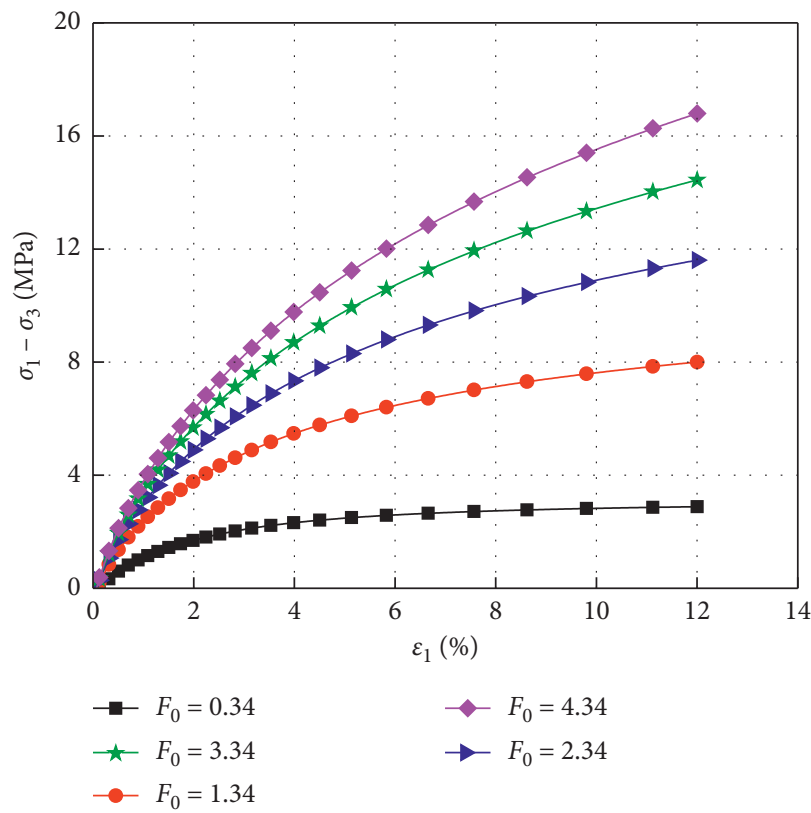

(a)

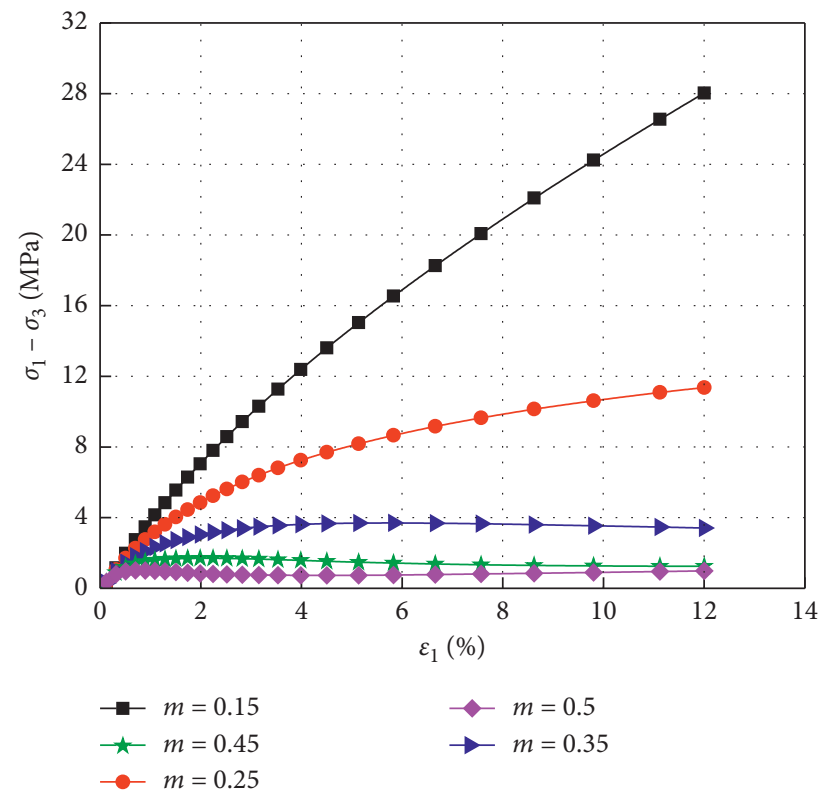

(b)

FIGURE 10: The relationship between the damage constitutive model of frozen sand and $F_{0}$ and $m$.

partial deviation between the revised theoretical curve and the experimental curve, the overall trend of the experimental curve and the revised theoretical curve is consistent and close to the actual engineering practice. The Weibull distribution parameters $F_{0}$ and $m$ affect the curve shape of the frozen sand damage constitutive model, and both are directly affected by the 


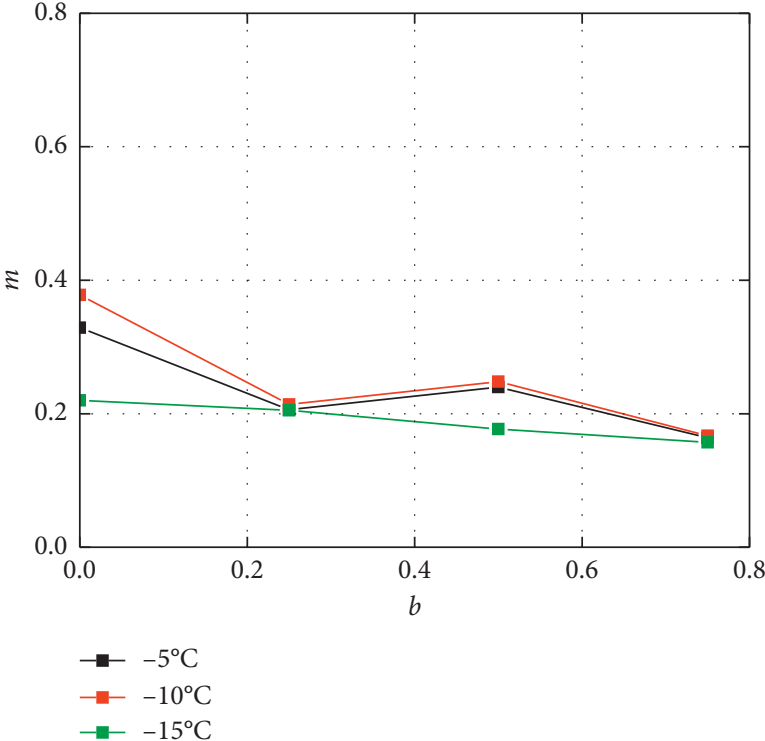

(a)

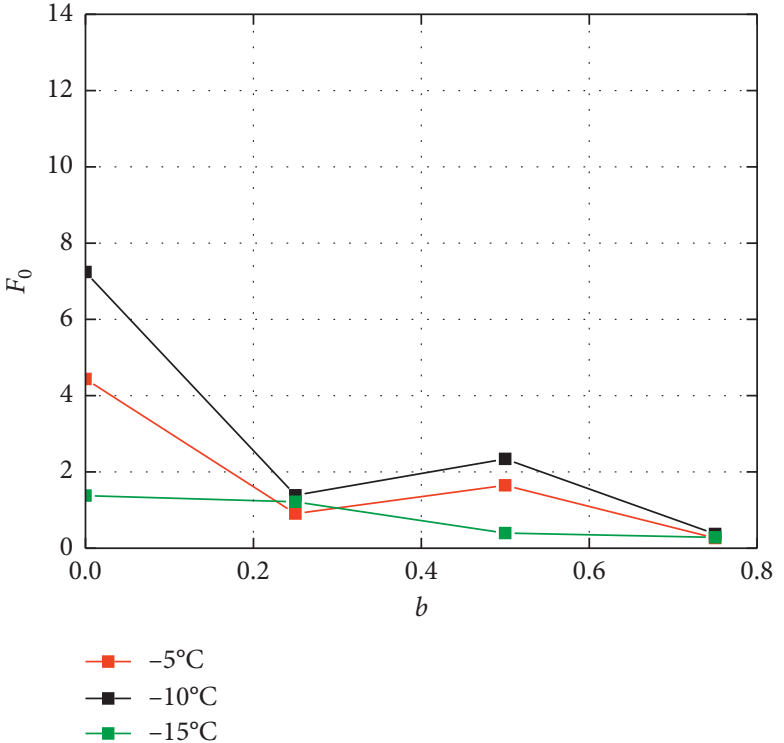

(b)

FIGURE 11: The relationship between the Weibull distribution parameters $F_{0}$ and $m$ with $b$ and $T$. In Figure 11, the linear fitting correlation coefficient $R^{2}$ of equation (31) is 0.96 , and the linear fitting correlation coefficient $R^{2}$ of equation (32) is 0.97. (a) The relationship between parameter $m$ to the temperature $T$ and the middle principal stress coefficient $b$. (b) Parameter $F_{0}$ variations with temperature $T$ and the middle principal stress coefficient $b$.

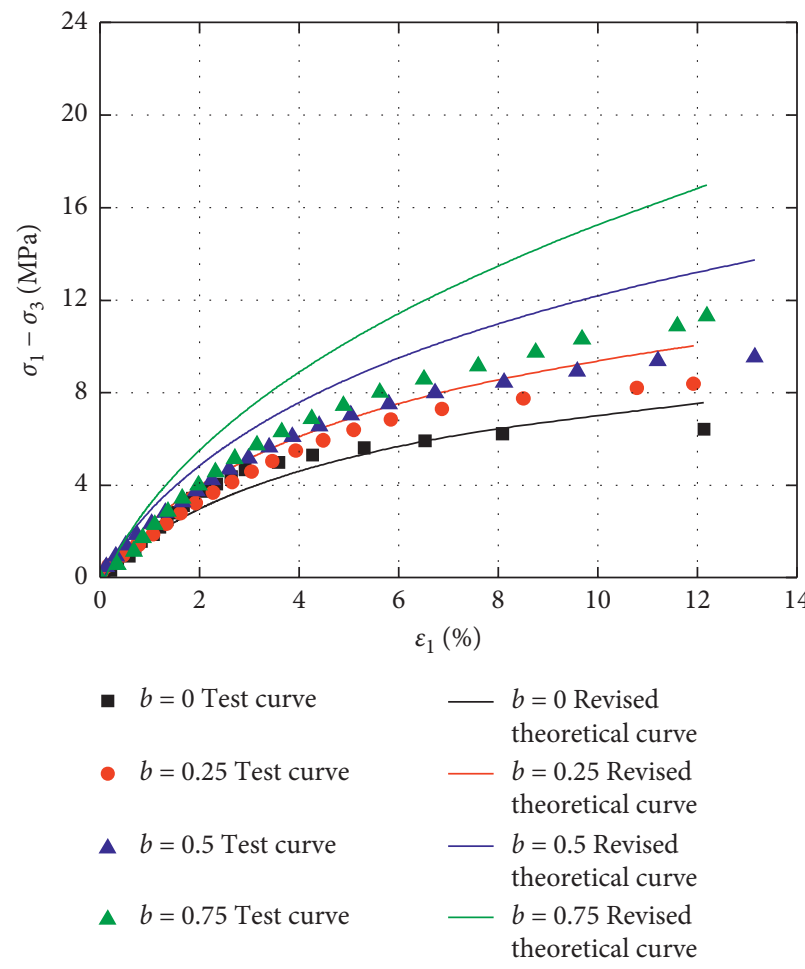

(a)

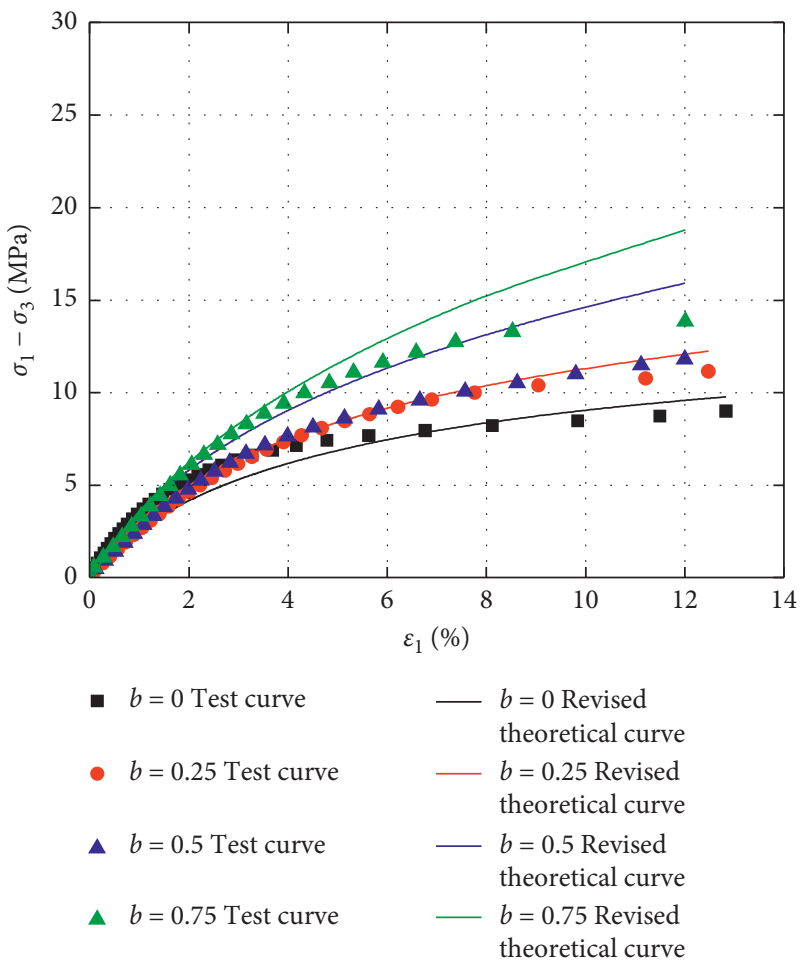

(b)

FIgURE 12: Continued. 


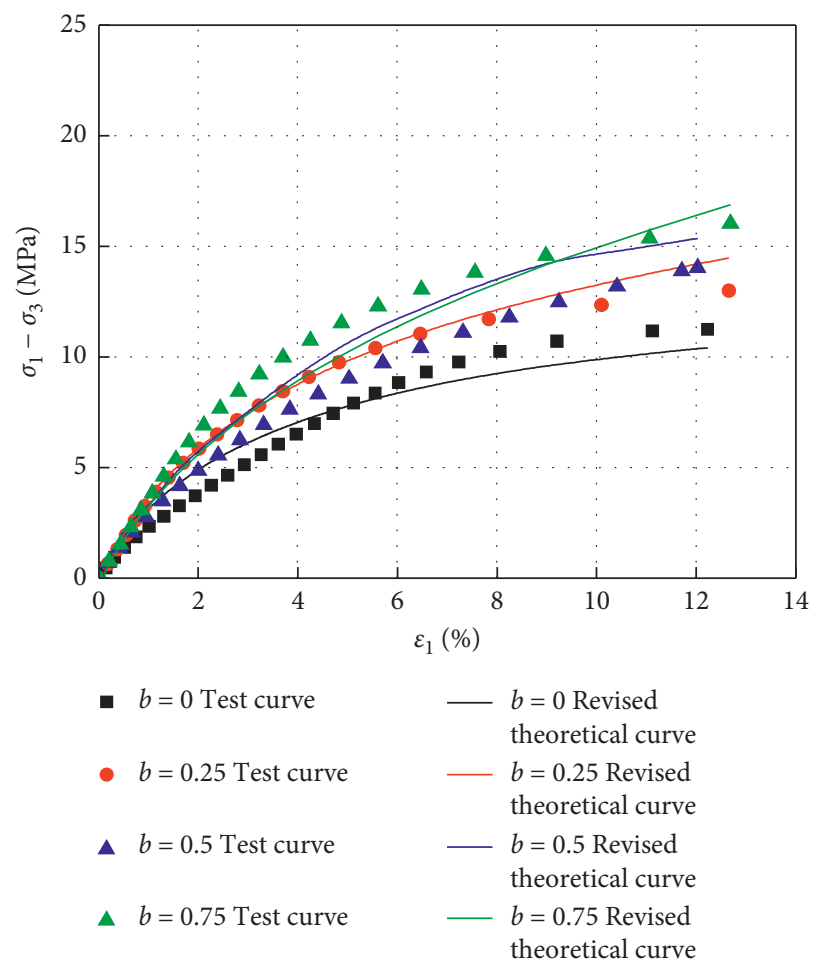

(c)

FiguRE 12: Test curve and modified theoretical curve at different temperatures and medium principal stress coefficients. (a) $-5^{\circ} \mathrm{C}$. (b) $-10^{\circ} \mathrm{C}$. (c) $-15^{\circ} \mathrm{C}$.

intermediate principal stress coefficient. This correction allows for the model to better reflect actual conditions compared to the existing model.

\section{Conclusion}

Based on previous studies [36], this paper assumes that the microelement strength distribution of frozen sand obeys the Weibull distribution [33-35]. This model uses the Drucker-Prager strength criterion as the microelement strength distribution variable, damage mechanics theory, and probability statistics and introduces the Weibull random distribution parameter $m$ and the relationship between $F_{0}$ and temperature and the medium principal stress coefficient. Therefore, a modified true triaxial damage constitutive model of frozen sand was derived. The established threedimensional damage constitutive model of frozen sand is simple with fewer parameters, easy determination of model parameters, and clear physical meaning. This modified model is also more convenient for practical engineering applications. The results can be summarized as follows:

(1) The modified damage constitutive model can well reflect the entire process of the failure of frozen sand under complex stress conditions.

(2) The model reflects that the strength of frozen sand is affected by temperature and the coefficient of intermediate principal stress. When the minimum principal stress is constant, as the temperature decreases and the coefficient of intermediate principal stress increases, the strength of frozen sand also increases.

(3) The model adequately reflects the influence of the damage caused by freezing temperatures and the complex stress state of the frozen sand during the failure process.

(4) The variations and physical meaning of Weibull distribution parameters $F_{0}$ and $m$ with temperature and intermediate principal stress coefficient were analyzed to obtain a modified frozen sand damage constitutive model. In addition, the trend of the model curve was consistent with the test result curve, with a better fit, indicating that the modified damage constitutive model is more reasonable compared to the current existing model.

\section{Data Availability}

The data used to support the findings of this study are available from the corresponding author upon request.

\section{Conflicts of Interest}

The authors declare that there are no conflicts of interest regarding the publication of this paper. 


\section{Acknowledgments}

This work was funded by the Open Project Fund of Key Laboratory of University of Fujian Province for Underground Engineering (no. KF-T18014)

\section{References}

[1] N. A. Tsytovich, The Mechanics of Frozen Ground, Science Press, Beijing, China, 1985.

[2] H. M. French, The Periglacial Environment, Essex, London, UK, 1996.

[3] X. Z. Xu, J. C. Wang, and L. X. Zhang, Physics of Frozen Soils, Science Press, Beijing, China, 2010.

[4] E. Chamberlain, C. Groves, and R. Perham, "The mechanical behaviour of frozen earth materials under high pressure triaxial test conditions," Géotechnique, vol. 22, no. 3, pp. 469-483, 1972.

[5] R. A. Bragg and O. B. Andersland, "Strain rate, temperature, and specimen size effects on compression and tensile properties of frozen soil," Engineering Geology, vol. 18, no. 12, pp. 35-46, 1981.

[6] A. M. Fish, "Strength of frozen soil under a combined stress state," in Proceedings of the Sixth International Symposium on Ground Freezing, Beijing, China, September 1991.

[7] S. Zhang, Y. Lai, Z. Sun, and Z. Gao, "Volumetric strain and strength behavior of frozen soils under confinement," Cold Regions Science and Technology, vol. 47, no. 3, pp. 263-270, 2007.

[8] Y. Lai, L. Jin, and X. Chang, "Yield criterion and elasto-plastic damage constitutive model for frozen sandy soil," International Journal of Plasticity, vol. 25, no. 6, pp. 1177-1205, 2009.

[9] J. Lemaitre, "Evaluation of dissipation and damage in metals," in Proceedings of the ICM Kyoto, Kyoto, Japan, August 1971.

[10] J. Lemaitre, "How to use damage mechanics," Nuclear Engineering and Design, vol. 80, no. 2, pp. 233-245, 1984.

[11] J. Lemaitre, "A continuous damage mechanics model for ductile fracture," Journal of Engineering Material and Technology, vol. 107, pp. 80-83, 1985.

[12] D. Krajcinovic, "Continuous damage mechanics revisited: basic concepts and definitions," Journal of Applied Mechanics, vol. 52, no. 4, pp. 829-834, 1985.

[13] T. Kawanoto, Y. Ichikawa, and T. Kyoya, "Deformation and fracturing behavior of discontinuous rock mass an damage mechanics theory," International Journal for Numerical Analysis Methods in Geomechanics, vol. 12, pp. 1-30, 1988.

[14] J. L. Chaboche, "Continuum damage mechanics: part I-general concepts," Journal of Applied Mechanics, vol. 55, no. 1, pp. 59-64, 1988.

[15] Z. Shen, "An elasto-plastic damage model for cemented clay," Chinese Journal of Geotechnical Engineering, vol. 15, no. 13, pp. 21-28, 1993.

[16] M. Brünig, "Numerical analysis and elastic-plastic deformation behavior of anisotropically damaged solids," International Journal of Plasticity, vol. 18, no. 9, pp. 1237-1270, 2002.

[17] M. Brünig, "An anisotropic ductile damage model based on irreversible thermodynamics," International Journal of Plasticity, vol. 19, no. 10, pp. 1679-1713, 2003.

[18] X. Zhao, H. Sun, and K. W. Lo, Damage Soil Mechanics, Tongji University Press, Shanghai, China, 2002.

[19] D. J. Celentano and J.-L. Chaboche, "Experimental and numerical characterization of damage evolution in steels," International Journal of Plasticity, vol. 23, no. 10-11, pp. 1739-1762, 2007.
[20] Z. Li, Theories and Application of Damage Mechanics, Science Press, Beijing, China, 2002.

[21] Y.-P. Yao and D. A. Sun, "Application of Lade's criterion to cam-clay model," Journal of Engineering Mechanics, vol. 126, no. 1, pp. 112-119, 2000.

[22] Y. P. Yao and A. N. Zhou, "Non-isothermal unified hardening model: a thermo-elasto-plastic model for clays," Géotechnique, vol. 63, no. 15, pp. 1328-1345, 2013.

[23] J. X. Ren and X. R. Ge, "Study of rock meso-damage evolution law and its constitutive model under uniaxial compression loading," Chinese Journal of Rock Mechanics and Engineering, vol. 20, no. 4, pp. 425-431, 2001.

[24] J. Qi and W. Ma, "A new criterion for strength of frozen sand under quick triaxial compression considering effect of confining pressure," Acta Geotechnica, vol. 2, no. 3, pp. 221-226, 2007.

[25] Y. M. Lai, Z. H. Gao, and S. J. Zhang, "Stress-strain relationships and nonlinear Mohr strength criteria of frozen sandy clay," Soils and Foundations, vol. 50, no. 1, pp. 45-53, 2010.

[26] Y. Lai, S. Li, J. Qi, Z. Gao, and X. Chang, "Strength distributions of warm frozen clay and its stochastic damage constitutive model," Cold Regions Science and Technology, vol. 53, no. 2, pp. 200-215, 2008.

[27] S. Y. Li, Y. M. Lai, S. J. Zhang et al., "An improved statistical damage constitutive model for warm frozen clay based on Mohr-Coulomb criterion," Cold Regions Science and Technology, vol. 57, no. 2-3, pp. 154-159, 2009.

[28] C. Tan, M. D. Yuan, Y. S. Shi, B.-s. Zhou, and H. Li, "Statistical damage constitutive model for cemented sand considering the residual strength and initial compaction phase," Advances in Civil Engineering, vol. 2018, Article ID 1410893, 2018.

[29] W. G. Cao, Z. L. Fang, and X. J. Tang, "A study of statistical constitutive model for softening and damage rocks," Chinese Journal of Rock Mechanics and Engineering, vol. 17, no. 6, pp. 628-633, 1998.

[30] W. G. Cao, M. H. Zhao, and C. X. Liu, "A study on damage statistical strength theory for rock," Chinese Journal of Geotechnical Engineering, vol. 26, no. 6, pp. 820-823, 2004.

[31] W. G. Cao, P. Li, and M. H. Zhao, "On statistical damage constitutive model and its parameters for rock based on normal distribution," Chinese Hydrogeology \& Engineering Geology, vol. 32, no. 3, pp. 11-14, 2008.

[32] J. Deng and D. Gu, "On a statistical damage constitutive model for rock materials," Computers \& Geosciences, vol. 37, no. 2, pp. 122-128, 2011.

[33] D. Krajcinovic and M. A. G. Silva, "Statistical aspects of the continuous damage theory," International Journal of Solids and Structures, vol. 18, no. 7, pp. 551-562, 1982.

[34] Z.-1. Wang, Y.-c. Li, and J. G. Wang, "A damage-softening statistical constitutive model considering rock residual strength," Computers \& Geosciences, vol. 33, no. 1, pp. 1-9, 2007.

[35] X.-1. Xu, M. Karakus, F. Gao, and Z.-z. Zhang, "Thermal damage constitutive model for rock considering damage threshold and residual strength," Journal of Central South University, vol. 25, no. 10, pp. 2523-2536, 2018.

[36] S. Huang, Q. Liu, A. Cheng, and Y. Liu, "A statistical damage constitutive model under freeze-thaw and loading for rock and its engineering application," Cold Regions Science and Technology, vol. 145, pp. 142-150, 2018.

[37] X. Pan, True Tri-axial Test on Stress-Strain Characteristics of Frozen Calcareous Clay, School of Civil Engineering and 
Architecture, Anhui University of Science and Technology, Huainan, China, 2018.

[38] W. C. Shi, J. G. Zhu, and H. L. Liu, "Influence of intermediate principal stress on deformation and strength of gravel," Chinese Journal of Geotechnical Engineering, vol. 30, no. 10, pp. 1449-1453, 2008.

[39] S. Liu, Constant Stress Ratio Loading Test of Frozen Sand and Study of Strength Criterion, School of Civil Engineering and Architecture, Anhui University of Science and Technology, Huainan, China, 2018.

[40] D. Zhang, E. L. Liu, X. Y. Liu et al., "A Damage constitutive model for frozen sandy soils based on modified MohrCoulomb yield criterion," Chinese Journal of Rock Mechanics and Engineering, vol. 37, no. 4, pp. 973-980, 2018.

[41] M. Zhang, C. S. Xu, X. L. Du et al., "True triaxial experimental research on shear behaviors of sand under different intermediate principal stresses and different stress paths," Shuili Xuebao, vol. 46, no. 9, pp. 1072-1079, 2015.

[42] X.-z. Ling, Z.-y. Zhu, F. Zhang et al., "Dynamic elastic modulus for frozen soil from the embankment on Beiluhe Basin along the Qinghai-Tibet Railway," Cold Regions Science and Technology, vol. 57, no. 1, pp. 7-12, 2009. 